\title{
Review of 250 Consecutive Cases of Vaginal Mesh Surgery for Genital Organ Prolapse
}

\author{
Salil Khandwala, MD, FACOG, ${ }^{1-3}$ Cheau Williams, MD, Elizabeth Rumschlag, MD, ${ }^{2}$ Jing Dai, PhD, ${ }^{3}$ \\ and Chaandini Jayachandran, $\mathrm{MS}^{1}$
}

\begin{abstract}
Objective: The aim of the study was to assess the success and complications of vaginal mesh surgery performed in 250 successive cases. Design: This study had a retrospective design. Materials and Methods: An analysis was performed of vaginal mesh surgery for complaints of stage II or greater pelvic organ prolapse (POP) from September 2008 to November 2010. A composite score that included subjective criteria of absence of a bothersome bulge, and objective criteria based upon Pelvic Organ Prolapse-Quantification (POP-Q) stage $<$ II and no re-interventions was used to assess treatment success. Results: Transvaginal mesh surgery was performed in 250 subjects (age: $64.40 \pm 11.26$ years, body mass index [BMI]: $28.96 \pm 5.38 \mathrm{~kg} / \mathrm{m}^{2}$ ) for POP. Ten $(4.0 \%)$ anterior mesh surgeries, $69(27.6 \%)$ posterior mesh surgeries, and 171(68.4\%) total mesh surgeries were performed in subjects with stage II or greater degrees of prolapse. Stage II prolapse was noted in $61(24.4 \%)$ subjects, 154 $(61.6 \%)$ had stage III, and $35(14.0 \%)$ had stage IV prolapse. The mean follow-up was 13 months, and 222 $(88.8 \%)$ patients were seen at least 12 months post-surgery. The composite success score was $89.2 \%$. Pure anatomic success based upon POP-Q< stage II was 94.1\%. Mean operative time was $119.71 \pm 41.58$ minutes. Mean intraoperative blood loss was $109.28 \pm 110.89 \mathrm{~mL}$. There were $12(5.4 \%)$ cases of mesh exposure in the vagina. There were no visceral injuries or mesh erosions. The incidence of de novo dyspareunia was $15.8 \%$. Conclusions: Transvaginal mesh surgery for POP is safe and effective, with few postoperative morbidities when performed by an experienced pelvic floor surgeon. ( J GYNECOL SURG 30:134)
\end{abstract}

\section{Introduction}

$\mathbf{U}$ nited States Census Bureau projections estimate that as many as 9,200,000 women in the United States will have prolapse by the year 2050, and, as may be expected, the demand for services to care for pelvic floor disorders is also estimated to increase by $45 \%$ by the year $2030 .{ }^{1}$ The lifetime risk of undergoing surgery for pelvic organ prolapse (POP) in the general female population up to the age of 85 years has recently been reported to be as high as $19 \%-20 \%{ }^{2}$

The cost of treating POP is also significant, because the direct costs of prolapse surgery were estimated to be $\$ 1,012$ million (in 1997). Furthermore, women with POP have an impaired quality of life (QoL), are more likely to be selfconscious, and are less likely to feel physically and sexually attractive than normal controls. ${ }^{3}$ Therefore, POP is not only a highly prevalent gynecologic condition, but its incidence also is exponentially increasing with escalating burden on decreasing health care resources. For all of these reasons, there is a need for surgical procedures that are minimally invasive with minimal complications and risk, and that offer low recurrence with high rates of long-term success.

Traditional non-mesh repairs have been hampered by high rates of recurrence. Weber et al. noted a high recurrence rate of $54 \%-70 \%$, especially of the anterior vaginal wall. ${ }^{4}$ Furthermore, Olsen et al. retrospectively observed that a woman has a $29 \%$ chance of re-operation for pelvic organ prolapse following an index surgery. ${ }^{5}$ This has led to an increasing push to incorporate synthetic mesh in POP repair. A recent meta-analysis encompassing 30 studies with 2653 patients calculated the objective success rates to be $87 \%-95 \%$ for several different commercial POP mesh kits. ${ }^{6}$

However, on July 13, 2011, the United States Food and Drug Administration (FDA) released a second Safety Communication in response to the reporting of more complications associated with transvaginal mesh placement. ${ }^{7}$ The FDA update stated that adverse events for POP mesh repair are not rare, as was previously reported, and brought

\footnotetext{
${ }^{1}$ Advanced Urogynecology of Michigan, P.C., Dearborn, MI.

${ }^{2}$ Oakwood Hospital and Medical Center, Dearborn, MI.

${ }^{3}$ Department of Obstetrics and Gynecology, Wayne State University, Detroit, MI.
} 
into question the relative effectiveness of meshed versus unmeshed repairs. Vaginal mesh surgery has its unique risks for complications, and the anatomic success may not necessarily indicate a functional or subjective improvement in outcomes.

This study reports on the use of a 50-50 blend mesh, Prolift $+\mathrm{M}^{\mathrm{TM}}$ (Ethicon Inc., Somerville, NJ) transvaginal mesh that contains poliglecaprone-25 knitted with polypropylene (PP-PG). The poliglecaprone-25 (Monocryl ${ }^{\mathrm{TM}}$ ) is absorbed after 3 months, leaving a lower burden of mesh in the vagina, decreasing from $57 \mathrm{~g} / \mathrm{m}^{2}$ to $31 \mathrm{~g} / \mathrm{m}^{2}$ (data on file: Ethicon Inc., Somerville, NJ). The pore size following absorption increases from $2.5 \mathrm{~mm}$ to $3.5 \mathrm{~mm}$. Preclinical studies have reported that the greater distance between pores resists the ability of "bridging fibrosis," thereby leading to a compliant and flexible scar tissue that mimics natural tissue mobility. ${ }^{8}$

This study was conducted in the first 250 successive patients who underwent the PP-PG vaginal mesh surgery for genital organ prolapse. The primary objective was to assess success based upon both subjective and objective criteria, and the secondary objective was to assess complications and also the impact of surgery on urinary incontinence, vaginal healing, and sexual function.

\section{Materials and Methods}

This is a retrospective study performed in the first 250 consecutive cases of POP managed with the PP-PG system from September 2008 to November 2010. The study was approved by the Institutional Review Board of Wayne State University. Ethicon Inc., the manufacturer of PP-PG mesh system, had no influence over the study, and no external funding was obtained for the study.

The inclusion criterion was having undergone a vaginal mesh surgery for complaints of stage II or greater prolapse. The exclusion criteria included not completing the validated questionnaires.

All definitions and descriptions conformed to the standards recommended by the International Continence Society. The anatomical outcome was assessed with the Pelvic Organ Prolapse-Quantification (POP-Q) score, which is described by the International Continence Society as a reliable and specific method to measure pelvic organ support.

The subjects completed validated questionnaires at preoperative and postoperative visits. The Pelvic Floor Distress Inventory-20 (PFDI-20) was used as a symptom-specific questionnaire for pelvic organ prolapse. The PFDI-20 subscores include Pelvic Organ Prolapse Distress Inventory (POPDI), ColoRectal Anal Distress Inventory (CRADI) and the Urogenital Distress Inventory-6 (UDI-6). ${ }^{9,10}$ The Medical Epidemiological and Social aspects of Aging (MESA) forms were used to assess associated urinary incontinence symptoms. ${ }^{11}$ Scores of the PFDI-20 were calculated in a simple additive fashion, in which a higher score indicated more distressful symptoms. The Dindo scale was used to classify postoperative complications. ${ }^{12}$

Success was defined by a composite score that comprised the following:

1. POP-Q stage $<$ II

2. PFDI Question 3 "Do you usually have a bulge or something falling out that you can see or feel in the vaginal area?" Answered No.

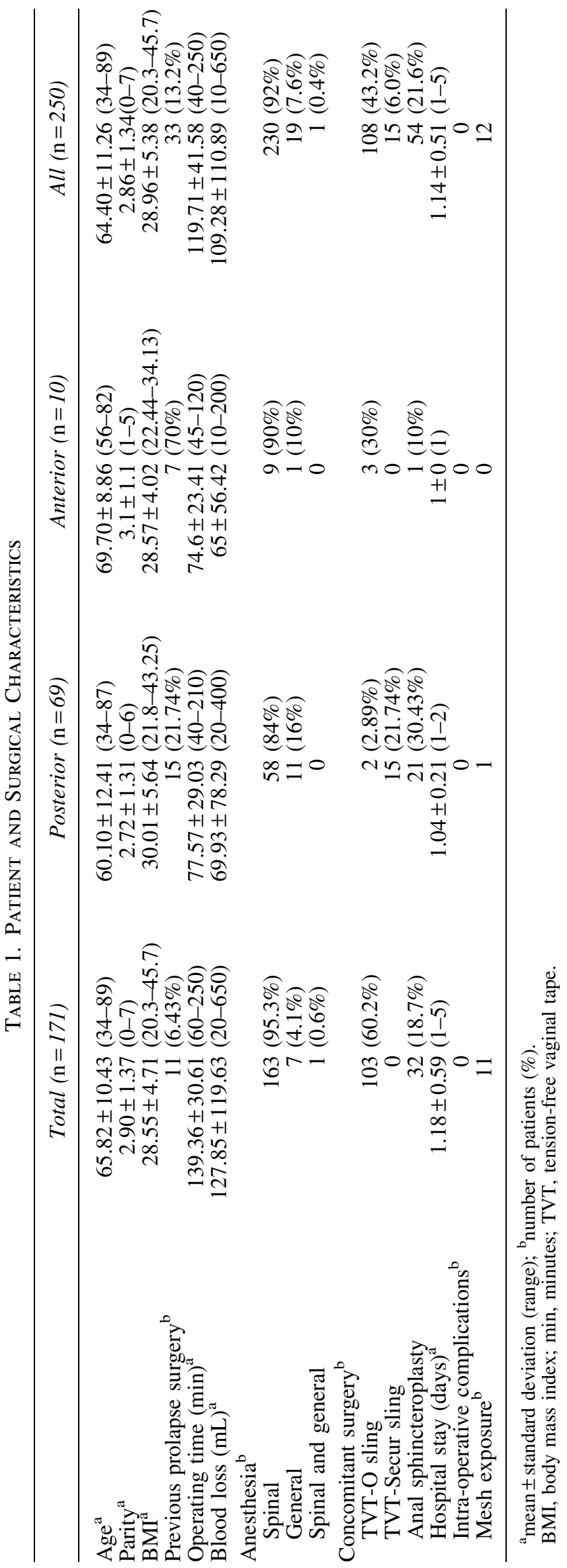


3. No reinterventions for POP such as pessary or surgery of either the treated or the untreated compartment.

In order for a subject to be considered to have had a successful outcome, that subject would have to have met each of the abovementioned criteria. Anatomic success (POPQ stage < II) but continued perception of a bothersome bulge constituted a failure.

All eligible subjects underwent a detailed urogynecologic history and examination including a POP-Q system assessment.

All subjects with an anterior vaginal wall prolapse, symptoms of urinary incontinence, or voiding dysfunction underwent urodynamic testing that was conducted in a standardized fashion. Occult stress urinary incontinence (SUI) was assessed by elevating the prolapsed anterior vaginal wall to simulate postsurgical repair. Intraoperative cystourethroscopy was performed in all subjects with anterior or total mesh surgeries or undergoing a concomitant sling procedure.

The surgical procedure was performed as per the technique described by the Trans-Vaginal Mesh group. ${ }^{13}$ For total vaginal mesh procedures, the mesh was kept intact as one piece and taken around an intact $2-3 \mathrm{~cm}$ apical bridge for post-hysterectomy vault prolapses. If the uterus was present, the mesh was cut into two pieces and the two halves were attached to the anterior and posterior aspect of the cervix, respectively. A permanent 2-0 prolene suture was used to anchor the mesh to the cervix.

The preoperative versus postoperative POPQ values were analyzed with the Statistical Package for the Social Sciences Version 20 (SPSS Inc. Chicago, IL) and were compared using paired sample $t$ test for all stages. The comparisons between preoperative and postoperative questionnaire scores were performed with the same method.

\section{Results}

Vaginal mesh surgery was performed in 250 patients from September 2008 to November 2010. This was the initial group of women to undergo this partially absorbable mesh procedure, and $222(88.8 \%)$ patients were seen at least 12 months post-surgery. The mean follow-up was $13.47 \pm 3.38$ months with a range of 7-31 months. Total vaginal mesh was performed in 171/250 (68.4\%) cases, anterior mesh surgery was performed in 10/250 (4\%), and posterior mesh surgery was performed in 69/250 (27.6\%). Detailed demographics and surgical characteristics are presented in Table 1. Stage II prolapse was noted preoperatively in 61/250 (24.4\%) sub- jects, stage III prolapse was noted in 154/250 (61.6\%), and stage IV prolapse was noted in 35/250 (14.0\%) subjects. Preoperative and postoperative POPQ information is listed in Tables 2 and 3.

The composite success score based upon determined parameters was $89.2 \%$. Anatomic success (POP-Q stage < II) was noted in $94.1 \%$ cases, whereas the lack of perception of a vaginal bulge was noted in $92.8 \%$. There was only one reoperation for prolapse, and this was the result of apical failure and necessitated a hysterectomy with sacrospinous ligament suspension. This patient had undergone a total vaginal mesh procedure.

All patients who had intraoperative cystoscopies demonstrated normal findings without any injury. The mean blood loss was $109.3 \mathrm{~mL}$ (standard deviation $\pm 111 \mathrm{~mL}$ ) and none of the patients received blood transfusions. Most patients were discharged home after an overnight stay, with a mean of $1.14 \pm 0.51$ days. Normal voiding function at discharge was noted in 226/250 (90.4\%) patients. The remaining 24 patients were sent home with a Foley catheter, which was successfully removed in 19 patients at the first attempt in the office on the 7 th postoperative day, as per protocol. Of these 24 patients sent home with a Foley catheter, $17(70.8 \%)$ patients had undergone a concomitant suburethral sling procedure. The remaining 5 patients failed the attempt, and were taught self-catheterization, which they eventually discontinued within 2 weeks. None of the patients had any lingering voiding dysfunction at their 1 year follow-up visit. Voiding dysfunction was defined as any subjective symptoms of difficulty urinating or elevated post-void residuals. Results of preoperative and postoperative validated questionnaire scores are listed in Table 4.

Mesh exposure as defined as mesh visible or palpable on vaginal exam or felt by patient or partner was noted in $5.4 \%$ $(12 / 222)$ subjects. Of these patients, 3 (25\%) underwent excision of the exposed mesh, 2 in the operating room (OR) and 1 in the office. The other patients $(75 \%)$ were managed expectantly. The mean area of exposure was $1.5 \mathrm{~cm}$. There were no erosions into the neighboring organs such as the bladder or the bowel. There were 5 cases of recurrent urinary tract infections (UTIs), and all these patients underwent a normal cystoscopy performed to rule out any mesh erosion into the bladder or the urethra. There was only 1 patient $(0.4 \%)$ with de novo stress urinary incontinence. The current authors did not look systematically for vaginal retraction during the period of the study. However, there was no change in the total vaginal length (TVL) noted from the preoperative to the postoperative values.

Table 2. Pre-Op and Post-op POPQ Stages

\begin{tabular}{|c|c|c|c|c|c|c|c|c|}
\hline \multirow[b]{2}{*}{$\begin{array}{l}P O P-Q \\
\text { Stage }\end{array}$} & \multicolumn{2}{|c|}{ Total } & \multicolumn{2}{|c|}{ Posterior } & \multicolumn{2}{|c|}{ Anterior } & \multicolumn{2}{|c|}{ All } \\
\hline & $\begin{array}{l}\text { Pre-op } \\
\mathrm{n}=171\end{array}$ & $\begin{array}{l}\text { Post-op } \\
\mathrm{n}=154\end{array}$ & $\begin{array}{c}\text { Pre-op } \\
\mathrm{n}=69\end{array}$ & $\begin{array}{c}\text { Post-op } \\
\mathrm{n}=60\end{array}$ & $\begin{array}{c}\text { Pre-op } \\
\mathrm{n}=10\end{array}$ & $\begin{array}{c}\text { Post-op } \\
\mathrm{n}=8\end{array}$ & $\begin{array}{l}\text { Pre-op } \\
\mathrm{n}=250\end{array}$ & $\begin{array}{l}\text { Post-op } \\
\mathrm{n}=222\end{array}$ \\
\hline 0 & 0 & 95 & 0 & 33 & 0 & 6 & 0 & 134 \\
\hline I & 0 & 52 & 0 & 23 & 0 & 2 & 0 & 77 \\
\hline II & 16 & 6 & 39 & 3 & 6 & 0 & 61 & 9 \\
\hline III & 121 & 1 & 30 & 1 & 3 & 0 & 154 & 2 \\
\hline IV & 34 & 0 & 0 & 0 & 1 & 0 & 35 & 0 \\
\hline
\end{tabular}

op, operative; POP-Q, Pelvic Organ Prolapse-Quantification. 


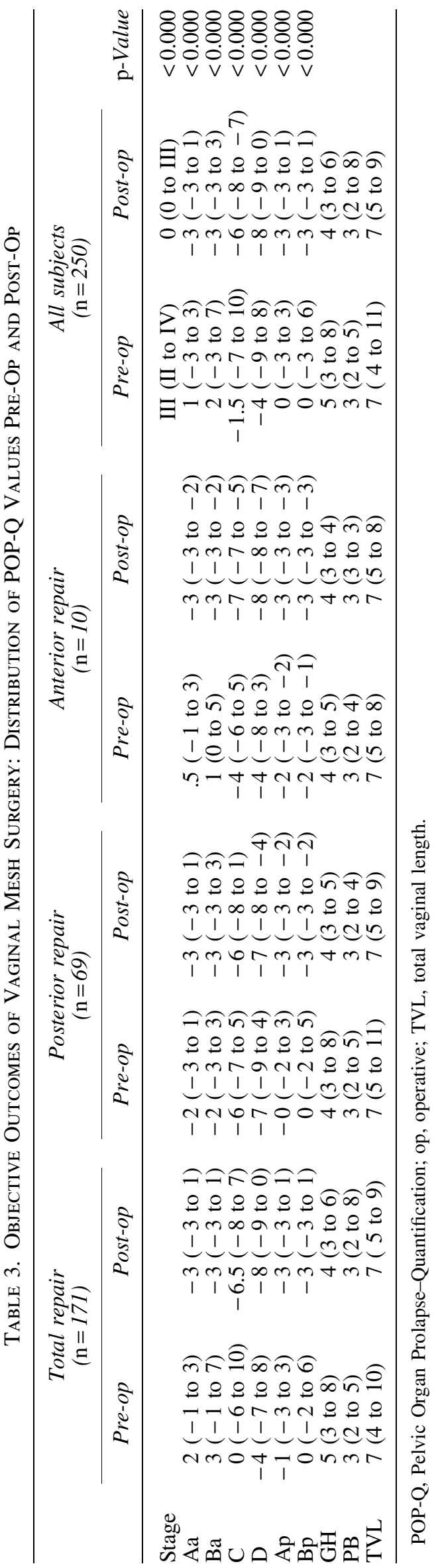

De novo dyspareunia was noted in 14/95 (14.7\%) patients. In this study, only 95 women were sexually active postoperatively. Twelve women, who had not been active preoperatively, became active postoperatively, and 1 of them complained of dyspareunia. If she is taken into account, the de novo dyspareunia rate is $15 / 95$, or $15.8 \%$.

The Dindo classification was as follows:

- Grade I: 27 subjects (15 de novo dyspareunia, 12 mesh exposures)

- Grade II: 10 subjects (5 UTIs, 5 straight catheterizations for 1 week)

- Grade IIIa: 1 subject (1 mesh revision in office)

- Grade IIIb: 4 subjects (2 mesh revisions in OR, 2 perineal revisions in OR)

- There were no Dindo Grade IV/V complications

The complications information is listed in Table 5.

\section{Discussion}

This retrospective cohort study of the first 250 successive patients treated with the second generation partially absorbable PP-PG system for pelvic organ prolapse shows good success in both the anatomic and functional domains. Definition of treatment success that includes improvement of vaginal bulge perception and absence of any new untoward side effects is more meaningful to a patient than just an anatomic cure. ${ }^{14}$ In this study, subjective improvement was noted in $92.8 \%$ cases.

A total of 222 patients were followed up for at least 12 months, providing at least a short- term evaluation. A comprehensive review article ${ }^{15}$ and a long term study ${ }^{16}$ have noted that most complications including mesh exposures become evident by 12 months. The reoperation rate for prolapse in this study was $0.4 \%(1 / 222)$ and is less than the $2.3 \%-4 \%$ reported in studies that provided a minimum 1 year follow-up. ${ }^{16,17}$ However, one of these studies ${ }^{16}$ involved $>500$ patients with a 3 year follow-up, unlike this one, which is only a 12 month short-term study. There were no injuries in this study. These results are consistent with most published reports on PP-PG mesh repair, in which the rates of visceral or vascular injuries ranged from 0 to $6.6 \%{ }^{18,19}$ The mesh exposure rate of $4.8 \%$ was similar to the $4 \%$ rate noted by Altman et al. ${ }^{20}$ de Landsheere et al., ${ }^{16}$ also in a large 500 case study followed for at least 3 years, noted a mesh exposure rate of only $2.7 \%$. Moreover, most of their exposures presented within the 1st year after surgery. This incidence is lower than the $10.3 \%$ for all synthetic mesh procedures reported by a systematic review by Abed et al. ${ }^{15}$ Nonetheless, it is comparable to the $3.4 \%$ reported rate after sacrocolpopexy in the review of Nygaard et al. ${ }^{21}$ Only $25 \%$ of the mesh exposures cases in the present study required a minor surgical intervention to correct them. Most were managed expectantly. This is similar to the report of Withagen et al. ${ }^{22}$

The incidence of anal sphincteroplasty in this series apparently seems high (21.6\%). In this series, anal sphincteroplasty was based on a patient's history of anal incontinence, the PFDI questionnaire pertaining to the bowel function, findings of anal sphincter disruption noted on clinical examination as a dove-tail sign, or a two finger rectal examination, and confirmed by anorectal manometry 
Table 4. Preoperative Versus Postoperative Validated Questionnaire Scores

\begin{tabular}{lcrl}
\hline & Pre-operative score & Post-operative score & p-Value \\
\hline POPDI $^{\mathrm{a}}$ & $41.3 \pm 26.7(0$ to 100$)$ & $8.1 \pm 13.2(0$ to 80$)$ & $<0.000$ \\
CRADI $^{\mathrm{a}}$ & $25.9 \pm 21.4(0$ to 89.3$)$ & $12.9 \pm 14.0(0$ to 87.5$)$ & $<0.000$ \\
UDI-6 $^{\mathrm{a}}$ & $37.1 \pm 27.6(0$ to 100$)$ & $14.2 \pm 16.9(0$ to 79$)$ & $<0.000$ \\
PFDI-20 SUMMARY $^{\mathrm{a}}$ & $104.1 \pm 61.6(0$ to 281.3$)$ & $35.2 \pm 34.5(0$ to 227.9$)$ & $<0.000$ \\
MESA STRESS $^{\mathrm{a}}$ & $30.0 \pm 28.9(0$ to 100$)$ & $14.8 \pm 19.2(0$ to 100$)$ & $<0.000$ \\
MESA URGE $^{\mathrm{a}}$ & $23.4 \pm 23.9(0$ to 88.9$)$ & $15.8 \pm 18.2(0$ to 83.3$)$ & $<0.000$ \\
\hline
\end{tabular}

${ }^{\mathrm{a}}$ Mean \pm standard deviation (minimum to maximum).

POPDI, Pelvic Organ Prolapse Distress Inventory; CRADI, Colo-Rectal Anal Distress Inventory; UDI-6, Urinary Distress Inventory-6; PFDI-20, Pelvic Floor Distress Inventory-20; MESA, Medical Epidemiological and Social aspects of Aging.

and anal ultrasound. Patients were counseled about the findings and surgery was based on the severity of symptoms and patient desire. It is being increasingly realized that women with other pelvic floor disorders may have a higher incidence of fecal incontinence $(35 \%-50 \%) .{ }^{23}$ Moreover, this is an underreported condition, and validated questionnaires unmask its presence as a bothersome symptom. ${ }^{24}$

There was only one case of de novo SUI $(0.4 \%)$. This is in contrast to the study by Cho et al., ${ }^{25}$ who noted a de novo SUI rate of $29.4 \%$. This is despite the fact that they, too, reduced the bulge with sponge sticks during preoperative urodynamics to assess for occult SUI. It is possible that their prolapse cases were very advanced, and hence may have been difficult to reduce accurately preoperatively.

The incidence of de novo dyspareunia in this study was $15.8 \%$. This is similar to the dyspareunia rate following traditional prolapse repair $(14.5 \%-36.1 \%)$, and a recent retrospective study reported a similar rate $(16.7 \%)$ following repair with the original polypropylene mesh. ${ }^{26}$ Although we did not specifically look at vaginal mesh retraction, there was no statistically significant decrease in the TVL from before surgery to the 12 month postoperative visit. Moreover, as PP-PG is a partially absorbable mesh, it may decrease the risk of bridging fibrosis, and, therefore, scar tissue formation. $^{27}$

TABle 5. Number of Intraoperative AND Postoperative COMPlications

\begin{tabular}{lr}
\hline Intraoperative complications & Number \\
\hline Bladder perforation & 0 \\
Rectal perforation & 0 \\
Ureteral injury & 0 \\
Blood transfusion & 0 \\
Postoperative Complications & \\
Recurrent urinary tract infection & 5 \\
Voiding difficulty & 7 \\
De novo stress urinary incontinence & 1 \\
De novo urge urinary incontinence & 1 \\
De novo fecal incontinence & 2 \\
Vaginal mesh erosion & 0 \\
Vaginal mesh exposure & 12 \\
Infection of mesh & 0 \\
Fistula & 0 \\
De novo dyspareunia & 15 \\
Recurrence of prolapse & 13 \\
\hline
\end{tabular}

The proportion of the combined (anterior and posterior) vaginal mesh procedures $(68 \%)$ to all mesh repairs was high in this study. This is mainly because the primary function of the posterior part of the total mesh was to support the vaginal apex to the sacrospinous ligaments more than to correct a rectocele. Also, several subjects in the study underwent a mesh hysteropexy, and this required a bilateral (anterior and posterior) cervical attachment for proper support. Moreover, care was taken to preoperatively identify small contralateral prolapses that in the past have resulted in failures of the unsupported contralateral compartment. ${ }^{28}$ This is similar to the findings of Withagen et al., ${ }^{19}$ who noticed a high risk of recurrence of the contralateral unsupported compartment following vaginal mesh surgery. Therefore, this study shows that if mesh is to be used, it should be planned correctly, and the contralateral apparently supported wall should be scrutinized carefully. Moreover, the value of the apex in total vaginal support is paramount. The anterior PP-PG mesh attaches laterally to the obturator internus and does not provide good DeLancey level I support as compared with the posterior arm, which attaches to the sacrospinous ligament.

Even though the PP-PG is no longer available, this study still helps in understanding the role of mesh in prolapse surgery. Moreover, the properties of PP-PG, such as the tensile strength, pore size, material type (type IA polypropylene), and weight are similar to those of the mesh systems still available today.

The strengths of this study are the enrollment of all successive patients who underwent the surgery during the 2 year time period, at least 1 year of follow-up, restriction to a single mesh kit, and the use of subjective and objective validated criteria to assess success, using validated outcome measures.

The weaknesses are those inherent to a retrospective design, mainly the lack of a control group. The POPQ assessments were performed by the primary surgeons (S.K., C.W.), and not by an independent observer. Although this could introduce an element of bias, the highly favorable patient subjective outcome supports the main results.

\section{Conclusions}

On July 13, 2011, the FDA released a second Safety Communication in response to the reporting of more complications associated with transvaginal mesh placement, stating that adverse events for POP mesh repair are not rare. ${ }^{7}$ 
There is growing consensus that vaginal mesh should neither be used in every prolapse case nor should it be abandoned, as it does have a place in the management of POP. ${ }^{29}$

There are several factors that determine success, failure, or risk of complications. The device is one that may be responsible for complications. It is hypothetically possible that certain local biochemical factors such as matrix metalloproteinase may play a role in the recurrence of prolapse and also vaginal healing. ${ }^{30}$ Surgical technique and experience have been increasingly shown to influence outcomes, and are among the most critical factors for success. ${ }^{31}$

Nevertheless, and most important, in an unclean environment, which the vagina presents, the implantation of foreign material exposes the patient to risks beyond that of autologous tissue repairs, which are by definition native to that patient.

\section{Disclosure Statement}

Dr. Khandwala has served as a consultant, was on the speaker's bureau, and has received grants from Ethicon Inc. Drs. Williams, Rumschlag, Dai, and Jayachandran declare that they have no conflicts of interest.

\section{References}

1. Wu JM, Hundley AF, Fulton RG, et al. Forecasting the prevalence of pelvic floor disorders in U.S. women: 2010 to 2050. Obstet Gynecol 2009;114:1278.

2. Smith FJ, Holman CD, Moorin RE, Tsokos N. Lifetime risk of undergoing surgery for pelvic organ prolapse. Obstet Gynecol 2010;116:1096.

3. Jelovsek JE, Barber MD. Women seeking treatment for advanced pelvic organ prolapse have decreased body image and quality of life. Am J Obstet Gynecol 2006;194:1455.

4. Weber AM, Walters MD, Piedmonte MR, Ballard LA. Anterior colporrhaphy: A randomized trial of three surgical techniques. Am J Obstet Gynecol 2001;185:1299.

5. Olsen AL, Smith VJ, Bergstrom JO, et al. Epidemiology of surgically managed pelvic organ prolapse and urinary incontinence. Obstet Gynecol 1997;89:501.

6. Feiner B, Jelovsek JE, Maher C. Efficacy and safety of transvaginal mesh kits in the treatment of prolapse of the vaginal apex: a systematic review. BJOG 2009;116:15.

7. U.S. Food and Drug Administration. FDA Safety Communication: UPDATE on Serious Complications Associated with Transvaginal Placement of Surgical Mesh for Pelvic Organ Prolapse. Online document at http://www.fda .gov/MedicalDevices/Safety/AlertsandNotices/ucm262435 .htm Accessed October 12, 2012.

8. Schug-Pass C, Tamme C, Sommerer F, et al. A lightweight, partially absorbable mesh (Ultrapro) for endoscopic hernia repair: experimental biocompatibility results obtained with a porcine model. Surg Endosc 2008;22:1100.

9. Barber MD, Walters MD, Cundiff GW, PESSRI Trial Group. Responsiveness of the Pelvic Floor Distress Inventory (PFDI) and Pelvic Floor Impact Questionnaire (PFIQ) in women undergoing vaginal surgery and pessary treatment for pelvic organ prolapse. Am J Obstet Gynecol 2006;194:1492.

10. Ubersax JS, Wyman JF, Shumaker SA, et al. Short forms to assess life quality and symptom distress for urinary incontinence in women: The Incontinence Impact Questionnaire and the Urogenital Distress Inventory. Neurourol Urodyn 1995; $14: 131$
11. Herzog AR, Diokno AC, Brown MB,Normolle DP, Brock BM. Two-year incidence, remission, and change patterns of urinary incontinence in non-institutionalized older adults. J Gerontol 1990;45:M67.

12. Dindo D, Demartines N, Clavien PA. Classification of surgical complications: a new proposal with evaluation in a cohort of 6336 patients and results of a survey. Ann Surg 2004;240:205.

13. Fatton B, Amblard J, Debodinance P, et al. Transvaginal repair of genital prolapse: Preliminary results of a new tension-free vaginal mesh (Prolift technique). A case series multicentric study. Int Urogynecol J Pelvic Floor Dysfunct 2007; 18:743.

14. Barber MD, Brubaker L, Nygaard I, et al. Defining success after surgery for pelvic organ prolapse. Obstet Gynecol 2009; 114:600.

15. Abed H, Rahn DD, Lowenstein L, et al. Incidence and management of graft erosion, wound granulation, and dyspareunia following vaginal prolapse repair with graft materials: a systematic review. Int Urogynecol J 2011;22:789.

16. de Landsheere L, Ismail S, Lucot J-P, Deken V, Foidart JM, Cosson M. Surgical intervention after transvaginal Prolift mesh repair: Retrospective single-center study including 524 patients with 3 years' median follow-up. Am J Obstet Gynecol 2012;206:83.e1.

17. Jacquetin B, Fatton B, Rosenthal C, et al. Total transvaginal mesh (TVM) technique for treatment of pelvic organ prolapse: a 3-year prospective follow-up study. Int Urogynecol J Pelvic Floor Dysfunct 2010;21:1455.

18. Altman D, Falconer C. Perioperative morbidity using transvaginal mesh in pelvic organ prolapse repair. Obstet Gynecol 2007;109:303.

19. Withagen MI, Vierhout ME, Milani AL. Does trocar-guided tension-free vaginal mesh (Prolift) repair provoke prolapse of the unaffected compartments? Int Urogynecol J Pelvic Floor Dysfunct 2010;21:271.

20. Altman D, Väyrynen T, Engh M E, Axelsen S, Falconer C, for the Nordic Transvaginal Mesh Group. Anterior colporrhaphy versus transvaginal mesh for pelvic-organ prolapse. N Engl J Med. 2011;364:1826.

21. Nygaard IE, McCreery R, Brubaker L, et al. Abdominal sacrocolpopexy: A comprehensive review. Obstet Gynecol 2004;104:805.

22. Withagen MI, Milani AL, den Boon J, et al. Trocar-guided mesh compared with conventional vaginal repair in recurrent prolapse: a randomized controlled trial. Obstet Gynecol 2011;117:242.

23. Jackson SL, Weber AM, Hull TL, Mitchinson AR, Walters MD. Fecal Incontinence in women with urinary incontinence and pelvic organ prolapse. Obstet Gynecol 1997;89:423.

24. Leigh RJ, Turnberg LA. Faecal Incontinence: The unvoiced symptom. Lancet 1982;1:134.

25. Cho MK, Kim CH, Kang WD, Kim JW, Kim S M, MD, Kim YH. Anatomic and functional outcomes with the Prolift procedure in elderly women with advanced pelvic organ prolapse who desire uterine preservation. J Minim Invasive Gynecol 2012;19:307.

26. Lowman JK, Jones LA, Woodman PJ, Hale DS. Does the Prolift system cause dyspareunia? Am J Obstet Gynecol 2008;199:707.e1.

27. Laschke MW, Häufel JM, Thorlacius H, Menger MD. New experimental approach to study host tissue response to surgical mesh materials in vivo. J Biomed Mater Res A 2005;74A:696. 
28. Khandwala S, Jayachandran C. Transvaginal mesh surgery for pelvic organ prolapse. Prolift $+\mathrm{M}$ : a prospective clinical trial. Int Urogynecol J 2011;22:1405.

29. Murphy M, Holzberg A, van Raalte H, Kohli N, Goldman HB, Lucente V, on behalf of the Pelvic Surgeons Network. Time to rethink: An evidence-based response from pelvic surgeons to the FDA Safety Communication: "UPDATE on Serious Complications Associated with Transvaginal Placement of Surgical Mesh for Pelvic Organ Prolapse' Int Urogynecol J 2012;23:5.

30. Vulic M, Strinc T, Tomic, et al. Difference in expression of collagen type 1 and matrix metalloproteinase- 1 in uterosacral ligaments of women with and without pelvic organ prolapse. Eur J Obstet Gynecol 2011;155:225.
31. Achtari C, Hiscock R, O'Reilly BA, et al. Risk factors for mesh erosion after transvaginal surgery using polypropylene (atrium) or composite polypropylene/polyglactin 910 (Vypro II) mesh. Int Urogynecol J Pelvic Floor Dysfunct 2005;16:389.

Address correspondence to: Salil Khandwala, MD, FACOG Advanced Urogynecology of Michigan, P.C. 18100 Oakwood Boulevard, Suite 213 Dearborn, MI 48124

E-mail: salil@augm.org 